\title{
16 Neue Governance-Konzepte für die Digitalisierung: Herausforderungen und Potentiale
}

\author{
Alexander Hamedinger
}

$\begin{array}{ll}\text { 1. Einleitung } & 344\end{array}$

2. Herausforderungen durch die Digitalisierung $\mathbf{3 4 5}$

$2.1 \quad$ Akteurlnnen, Institutionen und Akteurskonstellationen 345

$\begin{array}{lll}2.2 & \text { Legitimation } & 346\end{array}$

$\begin{array}{lll}2.3 & \text { Interaktionsprozesse } & 347\end{array}$

$\begin{array}{lll}2.4 & \text { Output von Governance } & 347\end{array}$

3. Auf der Suche nach neuen Governance-Konzepten 347

$\begin{array}{lll}3.1 & \text { Reflexive Governance } & 347\end{array}$

$\begin{array}{lll}3.2 & \text { Tentative Governance } & 349\end{array}$

$\begin{array}{llr}\text { 4. Fazit } & 350\end{array}$

$\begin{array}{ll}\text { Literatur } & 353\end{array}$

Alexandert Hamedinger

TU Wien, Forschungsbereich Soziologie (ISRA)

alexander.hamedinger@tuwien.ac.at

(C) Der/die Autor(en) 2021

M. Mitteregger et al. (Hrsg.), AVENUE21. Politische und

planerische Aspekte der automatisierten Mobilität,

https://doi.org/10.1007/978-3-662-63354-0_16 


\section{EINLEITUNG}

Unter dem Begriff „Governance“ versteht man grundsätzlich die Steuerung und Koordination von Handeln und umfasst im weiten Verständnis verschiedene Formen und deren Prozesse (vgl. Gailing/Hamedinger 2019). Diese reichen von hoheitlicher, d. h. staatlicher Top-downSteuerung und -Koordination bis zu Formen der Selbststeuerung ohne staatliche Akteurlnnen (Mayntz/Scharpf 1995, Blatter 2007). Dieses sozialtheoretisch fundierte Verständnis von Governance unterscheidet sich von einem engeren Verständnis, das den Wandel von staatlichen Steuerungs- und Koordinationsformen hervorhebt. Der Wandel, so die Argumentation, steht im Kontext der Veränderung von Akkumulationsregimen zunehmender Flexibilisierung. Der Staat als Teil der Regulationsweise wird modernisiert („Verwaltungsmodernisierung“) und unternehmerischer (Harvey 1989). Gleichzeitig öffnet er sich für die Mitwirkung von nichtstaatlichen Akteurlnnen an der politischen Entscheidungsfindung außerhalb der „klassischen“ Wege liberaler Demokratien. Public-private-Partnerships, Netzwerke, aber auch Partizipationsprozesse im Sinne der Bürgerbeteiligung sind Ausdrucksformen dieses Wandels von „government“ zu „governance“. Von Governance erhoffen sich Akteurlnnen aus Politik und Verwaltung eine Verbesserung der Effektivität und Legitimation von Steuerung und Koordination.

Vertreterlnnen der kritischen Planungstheorie und Stadtforschung (u. a. Yiftachel/Huxley 2000, Purcell 2009) wenden allerdings gegen eine Governance-Euphorie, die seit den 1980er Jahren in der europäischen Stadtentwicklung festzustellen ist, ein, dass unter anderem Partizipationsprozesse als Governance-Formen nicht zu einer erhofften Demokratisierung der Gesellschaft führen, sondern die Reproduktion von sozialer Ungleichheit unterstützen können. Governance befördere gar eine Neoliberalisierung (Deregulierungs- und Kommodifizierungspolitik) von Politik, Wirtschaft und Gesellschaft. Eine ähnliche Interpretation bringt Swyngedouw (2013) in die Debatte ein, wenn er von der politischen Ordnung der kapitalistischen Stadt heute spricht, die im Grunde genommen postpolitisch sei, weil darin die Aushandlung von tiefliegenden sozialen Konflikten vernachlässigt und die Einflussmöglichkeiten von starken Wirtschaftsakteurlnnen auf die Politik erhöht werden.

Befürworterlnnen einer solchen kritischen Argumentation verweisen weiterhin auf die Dominanz von „technokratischen Eliten“ in neueren Governance-Formen (z. B. in „policy networks“) sowie auf die fehlende Transparenz und demokratische Legitimation des Steuerns und Regierens durch Netzwerke oder Public-private-Partnerships (vgl. Jessop 2000, Zürn 2009, Hamedinger 2013). Zusammenfassend wird also an Governance - verstanden als neue Steuerungs- und Koordinationsform, an der Akteurlnnen aus unterschiedlichen gesellschaftlichen Sphären mitwirken - das teilweise Fehlen einer legitimatorischen Basis kritisiert. Unterstellt wird weiterhin, dass durch solche Governance-Formen Neoliberalisierung unterstützt und soziale Ungleichheit reproduziert wird. Vorwürfe, die natürlich für alle Formen der Steuerung und Koordination, auch der rein staatlichen und für neuere Governance-Konzepte, empirisch zu überprüfen wären.

Aus heutiger Sicht scheint diese Debatte um Government und Governance, Deregulierung und Re-Regulierung, Kommodifizierung und Rekommunalisierung, Öffnung und Schließung von Regulationsweisen (Reckwitz 2019) aktueller denn je. Dies hängt mit dem Kontext von Governance zusammen, der sich seit den 1980er Jahren in Europa ganz wesentlich verändert hat. Vier Aspekte sind dabei hervorzuheben:

1. Der Klimawandel, der sowohl eine Umkehr in den Zielen von räumlicher, gesellschaftlicher und ökonomischer Entwicklung (Stichwort: „degrowth“) und in den GovernanceFormen erfordert (Stichwort: neuer Munizipalismus). Wie schwierig das ist, zeigt Andrea Stickler (siehe Beitrag 18 in diesem Band) am Beispiel von Politiken der Verkehrswende auf. 
2. Der soziale Wandel, vor allem die zunehmenden sozioökonomischen Ungleichheiten, die sich unter anderem aus Struktureffekten am Arbeitsmarkt und Veränderungen in wohlfahrtsstaatlichen Systemen ergeben. Vor diesem Hintergrund sind Formen von Steuerung und Koordination gesucht, die den sozialen Zusammenhalt befördern und stabilisieren und eben nicht soziale Ungleichheit reproduzieren.

3. Der Wandel von politisch-administrativen Systemen und der liberalen Demokratien. Letztere stehen schon seit längerer Zeit vor der Herausforderung der Inklusion einer immer heterogener werdenden Gesellschaft. Interessensausgleich und Repräsentation dieser Vielfalt wird immer schwieriger. So wird der Anteil von nichtwahlberechtigten Bevölkerungsteilen in Städten immer höher (in Wien lag dieser Wert 2019 - nach Verlic/Hammer 2019 - bei 28 \% der Bevölkerung im Wahlalter), deren Inklusion in diese Systeme schwieriger.

4. Der technologische Wandel, der einer zielgerichteten Governance bedarf, um nicht negative Effekte auf die anderen genannten Handlungsbereiche (Gesellschaft, Umwelt, Demokratie) zu erzeugen (siehe Beitrag 19 von Dangschat in diesem Band). Gleichzeitig verändern sich durch die Digitalisierung Erscheinungsformen von Governance selbst. Banerjee et al. (Beitrag 20 in diesem Band) sprechen von „digital modes of governance“, die durch große Mengen an Daten möglich wären, und behaupten, dass die Regulierung von Datenströmen (deren Produktion und Verwendung) zusätzlich einen umfassenden, inklusiven Governance-Ansatz benötigt.

Welche Herausforderungen bringt der technologische Wandel - an dieser Stelle vor allem verstanden als Digitalisierung - für Governance-Strukturen- und -Prozesse nun mit sich? Mit welchen Governance-Konzepten sollte in Politik und Verwaltung auf diese Herausforderungen reagiert werden? Diesen Fragen wird im Folgenden kursorisch nachgegangen.

\section{HERAUSFORDERUNGEN DURCH DIE DIGITALISIERUNG}

Grundsätzlich lassen sich diese Herausforderungen entlang von vier Dimensionen beschreiben, durch welche Governance-Strukturen und -Prozesse gekennzeichnet sind. Diese Beschreibung ist allgemein gehalten, da eine detaillierte Beschreibung in konkreten Bereichen der Digitalisierung (z. B. bei Plattformen wie AirBnB oder beim automatisierten und vernetzten Verkehr, avV) jeweils eine vertiefte empirische Analyse voraussetzen würde.

\subsection{AKTEURINNEN, INSTITUTIONEN UND AKTEURSKONSTELLATIONEN}

Banerjee et al. (siehe Beitrag 20 in diesem Band) sprechen von "commercial inflections", die es zu regulieren gelte, da Akteurlnnen aus Politik und Verwaltung immer mehr von privatwirtschaftlich betriebenen Plattformen und der Sammlung von Daten abhängen, die eben oft von großen „Datenkonzernen“ (z. B. Microsoft, Google, Facebook) durchgeführt wird. Schulz und Dankert (2016) verweisen in ihrem Artikel über die "Governance by Things" auf Machtverschiebungen in den Akteurskonstellationen, die bisher für das Politikmachen ausschlaggebend waren: Sie sprechen von einer Verschiebung von Macht zugunsten von privaten Unternehmen, die Codes und Algorithmen entwickeln und implementieren. Auf städtischer Ebene finden sich insbesondere unter dem Titel der „Smart City“ Vorläufer der Digitalisierungsdebatte, die sich um die Rolle von Technologie in der Entwicklung von städtischen Ökonomien und Gesellschaften dreht. 
Hier zeigt sich aber, dass die digitale Transformation einen konfliktreichen Aushandlungsprozess zwischen starken Wirtschaftsakteuren (vor allem aus der IT-Branche), städtischer Politik und Verwaltung sowie mancherorts von sozialen Bewegungen beinhaltet (vgl. Bauriedl/Strüver 2018: 2) und technologiezentrierte und -deterministische Perspektiven zunehmend unter Rechtfertigungsdruck geraten. Die Breite des Akteursspektrums ist heute grundsätzlich ein wesentliches Kennzeichen von „urban governance“ (Cruz et al. 2019). Aber anders als manch kritischer Diskurs dies nahelegt, wirken Akteurlnnen aus städtischer Politik und Verwaltung an der Koproduktion der Smart City aktiv mit bzw. sind sie sogar wesentliche Treiber dieser Entwicklung.

Damit rückt das Government, das politisch-administrative System als Akteur in einer smarten, datengestützten räumlichen Entwicklung, wieder in den Mittelpunkt von Steuerungs- und Koordinationsvorhaben (s. den Beitrag von Dangschat in diesem Band). Allerdings sind Akteurlnnen aus Politik und Verwaltung mit den rasanten Entwicklungen rund um die Digitalisierung oft überfordert. Dies betrifft unter anderem ihr Wissen über die soziotechnischen Bedingungen und Effekte der Digitalisierung (siehe Beitrag 20 von Banerjee et al. in diesem Band), die juristischen Möglichkeiten der Einhegung negativer Effekte sowie die aus guten Gründen starren und zeitintensiven Prozesse der Kommunikation und der Entscheidungsfindung innerhalb der politischadministrativen Systeme. Die vertikal organisierte Aufbauorganisation dieser Systeme erschwert zudem eine rasche, ressortübergreifende Behandlung von neuen Problemen der Stadtentwicklung, die sich aus der Digitalisierung ergeben (z. B. im Fall von AirBnB). Eine weitere Herausforderung sprechen Schulz und Dankert (2006) an: Die Entwicklung von Codes und Algorithmen basiere auf explizitem Wissen, um Probleme zu lösen. Politische Entscheidungsfindungsprozesse und unsere alltäglichen Routinen dagegen basieren oft auf „tacit knowledge“, das nicht verbalisierbar ist. Diese Form von Wissen ist in politische Kulturen und gesellschaftliche Praktiken mit ihren jeweiligen sozialen Normen und Werten eingebettet und prägt unser Verhalten. Am Beispiel des automatisierten Fahrens führen Schulz und Dankert (ebd.: 8) zu Recht aus: „Testdrives with autonomous cars show that they are able to stick to traffic rules slavishly, but still cannot decide when to override rules reasonably [...]." Ein weiterer damit zusammenhängender Aspekt ist die Zeit- und Ortsgebundenheit von politischen und Planungskulturen.

Schließlich stellt sich die Frage, wer tatsächlich zu einem/r Akteurln in der "data-driven city" werden kann. Banerjee et al. thematisieren das bekannte Problem des „digital divide", das die ungleiche räumliche Verteilung digitaler Infrastrukturen sowie die ungleichen Kompetenzen und ökonomischen Ressourcen von sozialen Gruppen betreffe. Aufgrund der Geschwindigkeit von neueren Entwicklungen in der Digitalisierung könnten sogar die "digital natives“ zu "digital immigrants“ werden. Im Endeffekt verstärke der „digital divide“ die soziale Ungleichheit bzw. füge dieser neue Dimensionen hinzu. Zusammenfassend entsteht der Eindruck, dass die eingangs erwähnten Kritikpunkte an Governance - Machtverschiebungen zugunsten von privaten Unternehmen und technokratischen Eliten, Reproduktion von sozialer Ungleichheit - durch die Digitalisierung verschärft werden könnten, wenn nicht regulatorisch eingegriffen wird.

\subsection{LEGITIMATION}

Diese Veränderungen in Akteurskonstellationen sind dann problematisch, wenn dadurch bereits bestehende Legitimationsprobleme staatlicher Systeme verstärkt werden und/oder der Output von Governance im Bereich der Digitalisierung zu negativen Entwicklungen (eben u. a. zunehmende soziale Ungleichheit) führt. Schulz und Dankert (2006) sehen tatsächlich ein Legitimationsproblem, denn anders als bei der Entwicklung von Gesetzen, die in den klassischen Arenen liberaler Demokratien politisch diskutiert und entschieden werden, gibt es bei der Entwicklung der Codes keine politischen Debatten. Private Unternehmen müssen sich gar nicht politisch legitimieren. 


\subsection{INTERAKTIONSPROZESSE}

Die Prozesse der Aushandlung von unterschiedlichen Akteursinteressen und der politischen Entscheidungsfindung könnten durch die Digitalisierung verbessert werden (z. B. über interaktive Plattformen, Formen der E-Democracy) und damit einen Beitrag zur Demokratisierung leisten. Cruz et al. (2019: 11) betonen, dass digitale Technologien die Art und Weise verändert haben, wie Städte regiert werden (z. B. durch „city labs“). Allerdings, so Blühdorn und Kalke (2020), wird der Prozess der Demokratisierung häufig unterlaufen und nicht gestärkt: Die Aushandlung von konfligierenden und widersprüchlichen Interessen werde durch die digitale Revolution in "verengte Diskursräume“ (ebd.: 11) verlagert, ein wirklicher Diskurs, in dem es um „sachliche Überprüfung“ geht, komme in digitalen Foren nicht zustande: „In ganz ähnlicher Weise reduzieren auch digitale Filterblasen und Echokammern die Komplexität und installieren Denkblockaden und Denkverbote, die die gesellschaftliche Fragmentierung und Polarisierung befördern und den demokratischen Diskurs ersticken“ (ebd.: 11). Die digitale Revolution, oder weiter gedacht die „data driven city“, suspendiere die mündigen BürgerInnen.

\subsection{OUTPUT VON GOVERNANCE}

Schulz und Dankert (2006) meinen mit der Vorstellung der „Governance by Things“: „[...] We consider that which the loT (Internet of Things), code is law" paradigm [...] might enter the physical world with all its consequences. We call this the ,Governance by Things'." Mit anderen Worten: Es wird behauptet, dass Codes, welche die Funktionsweisen von Hard- und Software bestimmen, regulierend auf das konkrete menschliche Verhalten (neben anderen Regulierungsformen wie Gesetzen oder sozialen Normen) in der physischen Welt wirken. Schulz und Dankert (ebd.) verweisen gleich auf die offensichtliche Herausforderung, die mit dieser Vorstellung einhergeht. Für Regierungen entstehe das Problem, wie sie die Entwicklung von Codes durch private Unternehmen so beeinflussen können, dass sie legitimierten politischen Zielsetzungen (z. B. Klimaschutz, soziale Kohäsion, Gemeinwohl) entsprechen. Die Frage ist, ab welchem Zeitpunkt Codes zu einer öffentlichen Frage werden und damit öffentlich diskutiert werden müssten. Die Forderung von Schulz und Dankert (ebd.: 12) besteht darin, dass "Governance by Things" selbst eine Governance brauche, mit welcher die Codes „sozialisiert" werden.

\section{AUF DER SUCHE NACH NEUEN GOVERNANCE-KONZEPTEN}

Welche Governance-Konzepte wären nun geeignet, um mit diesen gravierenden Herausforderungen umgehen zu können? Hierbei werden im Folgenden zwei Ansätze diskutiert: „Reflexive Governance“ und „Tentative Governance“.

\subsection{REFLEXIVE GOVERNANCE}

Der Ausgangspunkt der steuerungstheoretischen Überlegungen von Voß und Kemp (2006) ist das Konzept der Nachhaltigkeit und die Frage, welche Form von Governance eine nachhaltige Transformation von Wirtschaft und Gesellschaft benötigt. Governance ist für Voß und Kemp ein Interaktionsprozess, in dem verschiedene AkteurInnen mit unterschiedlichen, vielfach konfligierenden Interessen aufeinandertreffen. Ergebnis von solchen Interaktionsprozessen sind dann unter anderem bestimmte „policies“ oder regulative Arrangements, insge- 
samt eine bestimmte Governance. Die Prozesse des gesellschaftlichen Problemlösens, die mit dem Begriff Governance beschrieben werden, umfassen die kollektive Problemanalyse und -definition, Zielformulierung und Evaluation von Lösungen sowie die Koordination von Handlungsstrategien.

Die Autoren argumentieren, dass Nachhaltigkeit aufgrund ihres grenzüberschreitenden Charakters (zwischen Problemen in Gesellschaft, Wirtschaft und Umwelt; zwischen Steuerungssektoren; zwischen AkteurInnen und Institutionen; zwischen Territorien) neue Formen der gesellschaftlichen Problembearbeitung braucht. Reflexive Governance sei ein geeigneter Weg, um auf diese komplexen Steuerungs- und Koordinationsherausforderungen reagieren zu können. Reflexivität bezieht sich auf die verschiedenen Formen von Governance, die angewandt werden, um bestimmte gesellschaftliche Probleme zu lösen: „Reflexive Governance thus implies that one calls into question the foundations of governance itself, that is, the concepts, practices and institutions by which societal development is governed, and that one envisions alternatives and reinvents and shapes those foundations" (ebd.: 4). Im Mittelpunkt steht die Reflexion über den Zyklus von Problemerzeugung und Problemlösung, über die Probleme von Steuerung und Koordination selbst, deren Effekte, die damit verbundenen Unsicherheiten und Ambivalenzen. Dies wird an anderer Stelle auch als „Second-Order Governance " bezeichnet (ebd.: 7). Eine solche Reflexion erfordere offene und lernorientierte Interaktionsprozesse, wie sie etwa für die transdisziplinäre Forschung oder partizipative Formen der Entscheidungsfindung bereits kennzeichnend sind.

Diese Komplexität von Problemen der Nachhaltigkeit hänge mit Folgendem zusammen:

1. Heterogenität von Elementen in der sozialökologischen Transformation, welche die Grenzen von spezialisiertem Expertenwissen aufzeigt, klassische Formen der Wissensproduktion in Frage stellt und neuere Formen benötigt, wie sie in der transdisziplinären Forschung angewandt werden.

2. Unsicherheiten, die mit der Unmöglichkeit der sicheren Vorhersage der Effekte und der Dynamiken von komplexen sozialökologischen Transformationen einhergehen. Dies erfordere adaptive Strategien und Strukturen, um aus Fehlern lernen zu können.

3. Pfadabhängigkeit: Zukünftige Entwicklungen seien durch historisch gewachsene Strukturen (z. B. Wertestrukturen, institutionelle Strukturen, Routinen der Problemlösung) beeinflusst, die nur schwierig und langsam verändert werden können. Entwicklungspfade können allerdings nicht vollkommen vorhergesagt werden. Durch unter anderem Szenarientechniken oder partizipative Modellierungen von Policies könnten einige alternative zukünftige Entwicklungen antizipiert und „lock-ins“ vermieden werden. Dazu komme, dass das Konzept der Nachhaltigkeit nicht wissenschaftlich objektiv bestimmt werden kann, sondern immer den Werturteilen unterschiedlicher Akteurlnnen (Wissenschaft, Politik, Planungspraxis, Zivilgesellschaft, Wirtschaft) unterliege. Ziele der Nachhaltigkeit seien damit quasi „,beweglich“. Die Konsequenz daraus ist für Voß und Kemp (ebd: 15f.): "Sustainability is thus and ambiguous and moving target that can only be ascertained and followed through processes of iterative, participatory goal formulation." Schließlich müssten bei der Umsetzung von Strategien einer nachhaltigen Transformation verschiedene Akteurlnnen, die wiederum unter anderem verschiedenen räumlichen Ebenen zuzuordnen sind (lokal, regional etc.), koordiniert werden. Insgesamt leiten Voß und Kemp (ebd.) daraus Anforderungen an Strategien reflexiver Governance ab: „integrated (transdisciplinary) knowledge production“, „adaptivity of strategies and institutions“, „anticipation of the long-term systemic effects of action strategies“, „iterative participatory goal formulation“, „interactive strategy development“. 
Dieses Konzept der Reflexive Governance weist einige Potentiale im Umgang mit den erwähnten Herausforderungen durch die Digitalisierung auf:

- In solchen offenen, lernorientierten und inklusiven Interaktionsprozessen könnte die Governance der "Governance by Things“ breit diskutiert werden. Dies würde Fragen zu den inhaltlichen Zielen einer solchen Governance und die Suche nach der besten Governance-Form für die Digitalisierung selbst betreffen (also wo braucht es staatliche Steuerung, Co-Governance, Selbstregulation oder Mischformen). Die Digitalisierung benötigt eine Reflexive Governance, damit nicht die oben angesprochenen negativen Effekte auf die Gesellschaft (soziale Ungleichheit), Demokratie (Entmündigung) und Umwelt entstehen.

- Das Wissen über die Bedingungen und Effekte der Digitalisierung ist auf unterschiedliche Akteursgruppen vor allem aus der Wirtschaft, aber auch aus der Zivilgesellschaft (NGOs), Politik und Verwaltung verteilt. Eine transdisziplinäre Wissensproduktion, welche diese unterschiedlichen Wissensformen und Handlungslogiken systematisch integriert, ist damit eine wesentliche Voraussetzung, um zielgerichtet steuern und koordinieren zu können. Für die Entwicklung von Strategien zum Umgang mit der Digitalisierung gilt daher: interaktiv und inklusiv. Ungleichgewichte in Machtverhältnissen müssten dabei bewusst angesprochen und in den Prozessen selbst austariert werden.

- Eine weitere Charakteristik der Digitalisierung spricht für eine Strategie einer Reflexive Governance: die Geschwindigkeit der technologischen Entwicklungen und die Komplexität der Auswirkungen dieser Entwicklungen (Unwissen über die nichtintendierten Folgen der Digitalisierung und Unvorhersehbarkeiten in Bezug auf den Kontext ; vgl. Beitrag 19 von Dangschat in diesem Band). Adaptive, lernende Strategien sowie partizipative Szenarienentwicklungen sind hier sicherlich geeignete Formen des Umgangs damit. Institutionelle Strukturen müssten adaptiver werden und regelmäßige Evaluationen und Monitoring der Effekte der Digitalisierung die Basis von Lernprozessen sein.

\subsection{TENTATIVE GOVERNANCE}

Der Ausgangspunkt für dieses Governance-Konzept sind für Kuhlmann et al. (2019) die Unsicherheiten und unerwarteten Risiken, die durch neue Entwicklungen in Wissenschaft und Technik entstehen können („emerging science and technology“). Zudem handle es sich dabei um Themenfelder, in welchen die Macht zur Beeinflussung von Governance-Lösungen auf unterschiedliche AkteurInnen verteilt sei. Um mit diesen kontextspezifischen Bedingungen umgehen zu können, brauche es laut Kuhlmann et al. (ebd.) einen flexiblen und deliberativen Governance-Ansatz, der einen guten Mittelweg zwischen der Herstellung notwendiger Stabilität einerseits und Flexibilität andererseits darstellt, also eine „tentative governance“: „We consider governance to be ,tentative "when it is designed, practiced, exercised or evolves as a dynamic process to manage interdependencies and contingencies in a non-finalizing way; it is prudent (e.g. involving trial and error, or learning processes in general) and preliminary (e.g. temporally limited) rather than assertive and persistent" (ebd.: 3). Es handelt sich um einen stark prozessorientierten Zugang zu Governance, der offen, lernorientiert und adaptiv ist sowie das Experimentieren mit verschiedenen Problemlösungen erlaubt. Eine solche Governance wird von Dangschat (Beitrag 19 in diesem Band) im Umgang mit avV gefordert.

Für Kulhmann et al. (2019) umfasst Governance verschiedene Arten des Regierens in unterschiedlichen Kontexten, wobei sie hervorheben, dass das hierarchische Steuern nicht mehr so gut funktioniert wie in der Vergangenheit. Koordination zwischen unterschiedlichen Akteurlnnen sei wichtiger geworden als das Steuern durch direkte Kontrollmechanismen. Es brauche aber beides, eine Tentative Governance als offener und flexibler Steuerungs- und Koor- 
dinationsansatz und "definitive forms of governance" (ebd.: 3), die stabiler und stark staatlich regulierend eingreifen, um bestimme Steuerungsziele erreichen zu können. Weiterhin unterscheiden die Autoren hinsichtlich der Effekte von Governance zwischen „intentional“ und „incidental". Eine definitive Form von Governance könnte zu einer Tentative Governance werden, wenn unvorhersehbare Anpassungen im Steuerungsprozess notwendig werden. Tentative Governance könne vor allem in Kontexten gefunden werden, in denen "weiche“ Formen der Regulation (etwa Benchmarking) und "harte" nebeneinander bestehen (die Autoren nennen dies „hybrid arrangements“). Tentative Governance werde dabei oft an den „Schatten der Hierarchie" angebunden, d. h. mit harten Steuerungsformen (u. a. Gesetzgebung) verknüpft. Tentative Governance überschneide sich mit anderen Formen von Governance, die in der Literatur bisher genannt wurden, so etwa mit Reflexive und Adaptive Governance. Allerdings sei ihr Konzept der Tentative Governance breiter als der Ansatz der Reflexive Governance, so Kuhlmann et al. (ebd.), da über beides reflektiert wird: die kognitiven Fundamente von Governance selbst sowie über deren Funktionsweise. Die meisten Berührungspunkte sehen sie zu einer „experimentalist governance", die auf Flexibilität, Offenheit, Lernen und Reversibilität beruhe. Ein Vorteil ihres Konzeptes der Tentative Governance bestehe weiterhin in der Möglichkeit seiner Anwendung in verschiedenen räumlichen, zeitlichen und sozialen Kontexten. Sie betrachten es als Heuristik, um verschiedene idealtypische Prozesse der Herstellung oder des Wandels sozialer Ordnung aufdecken zu können.

Auch das Konzept der Tentative Governance weist einige Potentiale im Umgang mit der Digitalisierung auf:

- Wie die Autoren selbst betonen, besteht der Mehrwert von Tentative Governance darin, deutlich zu machen, dass im Kontext von Innovation und technologischer Entwicklung Governance so konzeptualisiert werden muss, dass unrealistische Steuerungshoffnungen vermieden werden. Unsicherheiten, Komplexität und Dynamik erfordern unter anderem solche Formen von Governance.

- Mehr noch als im Konzept der Reflexive Governance wird betont, dass stark prozessorientierte und offene Formen von Governance an andere, stärker regulatorische und hierarchische Formen von Steuerung angebunden werden müssen („Schatten der Hierarchie“). Für den Umgang mit den Bedingungen und Folgen der Digitalisierung scheint dies als essentiell, da einige Entwicklungen im Bereich von Technologie und Innovation (z. B. Plattform-Urbanismus) ein regulatorisches Framing benötigen. Ein Mix von harter und weicher Steuerung und Koordination ist mit diesem Konzept möglich.

- Tentative Governance kann als Forschungsstrategie in verschiedenen Kontexten angewendet werden. Das Konzept ist hilfreich, um die kontextspezifischen Herausforderungen der Digitalisierung (etwa in unterschiedlichen Stadttypen) herausarbeiten zu können.

\section{FAZIT}

Grundsätzlich ist mehr empirische Forschung zum Zusammenhang von (Urban) Governance und Digitalisierung notwendig: „Advances in technology, the cost reduction of specialized hardware, and the open source and open data movements are redrafting the rules of the game for public services, community engagement, and urban entrepreneurship. [...] However, our understanding of the implications of these changes is still meager" (Cruz et al. 2019: 11). Wie 
die Digitalisierung in unterschiedlichen Themenfeldern der Stadtentwicklung (Mobilität, Energie etc.) auf historisch gewachsene Governance-Systeme wirkt und wie eine Governance der Governance der Digitalisierung (im Grunde eine Reflexive Governance) etabliert werden kann, ist noch zu wenig empirisch beleuchtet worden. Für eine Reflexive Governance braucht es aber auf jeden Fall eine offene Diskussion zwischen „Technologie-EnthusiastInnen“, die nur die Vorteile der "data driven city“ sehen, und „Technologie-KritikerInnen“, die (ausschließlich) eine Verstärkung der sozialen Ungleichheit und eine Erosion der Demokratie befürchten (vgl. ebd.). Zudem muss die Digitalisierung in verschiedenen Bereichen der Stadtentwicklung die Komplexität und Heterogenität von Städten berücksichtigen. Dafür braucht es wiederum an den jeweiligen Kontext angepasst Formen von Governance, wie sie im Konzept der Tentative Governance gefordert wird.

Reflexive und Tentative Governance haben also einige Potentiale hinsichtlich der Governance der Digitalisierung. Ein wesentlicher Unterschied besteht allerdings in ihrem Ausgangspunkt: Während im Konzept der Reflexive Governance das Ziel von Steuern und Koordinieren die Nachhaltigkeit ist, geht es bei der Tentative Governance um die Suche nach neuen Governance-Formen im Kontext von Innovation und rasantem technologischem Wandel. Das Konzept der Tentative Governance bleibt also in Bezug auf das Steuerungsziel relativ neutral. Darauf verweisen Kuhlmann et al. (2019) selbst. Aus analytischer Sicht ist das verständlich, denn damit ist es möglich, verschiedene - wie sie sagen - Idealtypen der Herstellung sozialer Ordnung verstehen zu können. Aus der Sicht von Akteurlnnen aus Politik, Verwaltung, Wirtschaft und Zivilgesellschaft könnte dies allerdings wenig hilfreich sein, da es innen zuerst um die Suche nach Zielen der Stadtentwicklung gehen müsste und erst in einem nächsten Schritt um die Suche nach Governance-Formen, um diese Ziele umsetzen zu können. Aufgrund der derzeitigen multiplen Krise (vgl. Brand 2014), die Wirtschaft, Gesellschaft, Umwelt und Demokratie betrifft, braucht es sicherlich Ziele von Governance, die in Richtung sozialökologische Transformation oder Nachhaltigkeit gehen.

Eine Gemeinsamkeit und gleichzeitig eine Kritik an beiden Governance-Konzepten ist die weitgehende Vernachlässigung von Fragen der Legitimation. Dies ist quasi ein alter Vorwurf (siehe Einleitung) gegenüber der Governance, dem in den ausgeführten Konzepten jedoch nicht begegnet wird. Fragen der Legitimation von in beiden Konzepten geforderten lern- und prozessorientierten Governance-Formen müssen allerdings gestellt werden, da damit aufgedeckt werden kann, welche Akteurlnnen exkludiert sind, wer wirklich ein Mitwirkungs- und Mitspracherecht an interaktiver Strategieentwicklung und transdisziplinärer Wissensproduktion hat. Im Kern geht es um das Aufdecken der demokratiepolitischen Qualität solcher neuer Governance-Konzepte. Die Anbindung an klassische Arenen der demokratischen Entscheidungsfindung, wie sie im Konzept der Tentative Governance zumindest angesprochen wird, wird dabei nicht ausreichen. Für Reflexive und Tentative Governance müssen neue Formen der Legitimation gefunden werden. Ansonsten werden demokratiepolitische Defizite und die Legitimationskrise der politisch-administrativen Systeme reproduziert oder gar verstärkt.

Ein weiterer kritischer Punkt betrifft den Output von Governance, vor allem die Steuerung des Verhaltens unterschiedlicher AkteurInnen durch Maßnahmen der Digitalisierung (etwa durch avV in Bezug auf das Mobilitätsverhalten). Schulz und Dankert (2006) haben bereits die rechtlichen Problematiken des Steuerns durch Codes und Algorithmen deutlich gemacht, während Blühdorn und Kalke (2020) auf die negativen demokratiepolitischen Effekte der Digitalisierung verweisen. Am Beispiel des digitalen „Nudging“, das ein „Anstupsen“ des Verhaltens durch digitale Anreize in eine gewünschte Richtung bedeutet, könnten und müssten Fragen der Governance und der Demokratie diskutiert werden. Das Nudging im Bereich des Konsumverhaltens (z. B. des Energiekonsums) wirft nicht nur Fragen hinsichtlich der digitalen Verhaltensüberwachung auf, sondern auch des Einschränkens von Freiheitsrechten. Handelt es sich beim Nudging um ein Manipulationsinstrument oder erhöht sich damit die Wahlfreiheit des Subjekts 
wie es von Thaler und Sunstein (2019) behauptet wird? Sind die Ziele des Nudging im demokratischen Prozess breit und transparent ausgehandelt worden? Sind diese Ziele für KonsumentInnen sichtbar? Wie werden die privaten Unternehmen, die das Auswahldesign für die Verhaltenssteuerung konzipieren, politisch überwacht?

Es sind also noch einige Fragen theoretisch und empirisch zu klären, bevor unbedacht auf zunächst gut klingende Governance-Konzepte aufgesprungen wird. Aber gibt es vielleicht zumindest ein Steuerungsparadigma, dass als übergeordnetes zukünftiges Leitbild der Governance der Digitalisierung herhalten kann? Reckwitz (2019) zeichnet einen möglichen Mittelweg zwischen den beiden bisherigen dominanten Paradigmen, dem sozialkorporatistischen Paradigma und dem apertistischen Liberalismus, einem Regulierungs- und einem Dynamisierungsparadigma. Die für den apertistischen Liberalismus kennzeichnenden Verflechtungen zwischen Politik und Ökonomie (etwa in Form von Public-private-Partnerships), Maßnahmen der Deregulierung und Privatisierung sowie die zunehmende Vielfalt und Anzahl von Steuerungsakteuren sind sicherlich Kernelement des Wandels von Government zu Governance. Die negativen Effekte dieses Liberalismus sind laut Reckwitz (vgl. ebd.: 271ff.) enorm: Die mangelnde soziale und staatliche Rahmung von Märkten habe unter anderem zu einer Verstärkung von sozialen Ungleichheiten geführt, die Verschiebung von Macht zugunsten von internationalen Organisationen, des Rechtswesens und implizit starken Wirtschaftsakteuren hat den Prozess der Entdemokratisierung vorangetrieben.

Ein weiterer Treiber des Vertrauensverlustes in liberale Demokratien seien unter anderem die digitalen Medien, die Formen von Meinungs- und Willensbildung erzeugen, mit denen die traditionellen Institutionen nicht Schritt halten können. Reckwitz beschreibt nun ein neues Steuerungsparadigma, das diesen Neoliberalismus ablösen soll, den „regulativen oder einbettenden Liberalismus“ (ebd.: 285). Dieser beinhalte Regulierungen im Sinne sozialer Ordnungsbildung, reagiert regulierend auf die neuen sozialen und kulturellen Fragen (u. a. durch staatliche Regelsetzungen für Märkte und zum Schutz von individuellen Rechten) und behält eine liberale Grundlage bei, welche die Dynamik von Märkten und der Globalisierung nutzt und in neue Rahmenbedingungen einbettet. Für die Frage der Governance ist das Staatsverständnis vielsagend: „Der Staat des regulativen Liberalismus ist wieder ein aktiverer Staat - allerdings neuen Typs, der sich vom steuernden Nationalstaat des Sozial-Korporatismus deutlich unterscheidet [...]" (ebd.: 290). Und weiter heißt es: „Aus der Sicht des liberalen Regierens kann aber eine Steuerung gesellschaftlicher Prozesse immer nur indirekt verlaufen, mithilfe von Anreizen und Hindernissen erfolgen und muss mit nichtintendierten Folgen des eigenen Handelns rechnen“ (ebd.: 292).

Für die Governance der Digitalisierung könnte das von Reckwitz vorgeschlagene Steuerungsparadigma des regulativen Liberalismus durchaus taugen, da die negativen Effekte der Digitalisierung sozial und ökologisch regulierend bearbeitet werden könnten, ohne unternehmerische Innovationen und die für technologische Entwicklungen wichtige Dynamik von Märkten zu untergraben. Wenn Steuerung und Koordinieren allerdings hauptsächlich durch Anreize funktionieren soll, dann gelten die gleichen demokratiepolitischen Vorbehalte wie beim Nudging. Dieser Sachverhalt müsste auf der Suche nach einem neuen Steuerungsparadigma im Bereich der Digitalisierung von allen relevanten Akteurlnnen breit diskutiert werden. 


\section{LITERATUR}

Bauriedl, S., und A. Strüver (Hrsg.) 2018. Smart City - kritische Perspektiven auf die Digitalisierung in Städten. Bielefeld: transcript.

Blatter, J. 2007. Governance - theoretische Formen und historische Transformationen. Baden-Baden: Nomos.

Blühdorn, I., und K. Kalke. 2020. „Entgrenzte Freiheit. Demokratisierung im ökologischen Notstand?“, IGN Interventions, Febr. 2020, hg. v. Institut für Gesellschaftswandel und Nachhaltigkeit (IGN), WU Wien. www.wu.ac.at/fileadmin/wu/d/i/ign/IGN_Interventions_Feb_2020.pdf (31.6.2020).

Brand, U. 2014. „Sozial-ökologische Transformation“, in Kurswechsel 2/2014, 7-18.

Cruz, N. F. da, P. Rode, und M. McQuarrie. 2019. „New Urban Governance: A Review of Current Themes and Future Priorities“, in Journal of Urban Affairs (41) 1, 1-19.

Gailing, L., und A. Hamedinger 2019. „Neoinstitutionalismus und Governance“, in ARL Reader Planungstheorie, hg. v. T. Wiechmann, Band 1. Berlin: Springer, 167-178.

Hamedinger, A. 2013. „Governance, Raum und soziale Kohäsion - Aspekte einer sozial kohäsiven stadtregionalen Governance“, unveröffentl. Habilitationsschrift, TU Wien.

Harvey, D. 1989. „From Managerialism to Entrepreneurialism: Formation of Urban Governance in Late Capitalism“, in Geografisker Annaler 71(B), 3-17.

Jessop, B. 2000. „Governance Failure“, in The New Politics of British Local Governance Stoker, hg. v. G. Stoker. London: MacMillan Press, 11-32.

Kuhlmann, S., P. Stegmaier und K. Konrad 2019: „The tentative governance of emerging science and technology - A conceptual introduction“, in Research Policy (48) 5, 1091-1097.

Mayntz, R., und Scharpf, F. W. (Hrsg.) 1995. Gesellschaftliche Selbstregelung und Politische Steuerung. Frankfurt am Main/New York: Campus.

Purcell, M. 2009. „Resisting Neoliberalization: Communicative Planning or Counter-Hegemonic Movements?“, in Planning Theory (8) 2, 140-165.

Reckwitz, A. 2019. Das Ende der Illusionen. Politik, Ökonomie und Kultur in der Spätmoderne. Frankfurt am Main: Suhrkamp.

Schulz, W., und K. Dankert 2016. „,Governance by Things“ as a challenge to regulation by law“, in Internet Policy Review (5) 2, 1-20.

Swyngedouw, E. 2013. „Die postpolitische Stadt“, in sublurban - Zeitschrift für kritische Stadtforschung (1) 2, 141-158. DOI: 10.36900/suburban.v1i2.100.

Thaler, R. H., und C. R. Sunstein 2019. Nudge. Wie man kluge Entscheidungen anstößt, 14. Aufl. Berlin: Ullstein.

Verlic, M., und K. Hammer 2019. „Mind the Gap - Achtung demokratische Beteiligung“. A\&W blog, 31.10.2019. https://awblog.at/achtung-demokratische-beteiligung/ (9.9.2020).

Voß, J. P., und R. Kemp 2006. „Sustainability and Reflexive Governance: Introduction“, in Reflexive Governance for Sustainable Development, hg. v. J. P. Voß, D. Bauknecht und R. Kemp. Cheltenham: Edgar Elgar, 3-30.

Yiftachel, O., und M. Huxley 2000. „Debating Dominance and Relevance: Note on the ,Communicative Turn“ in Planning Theory“, in Journal of Planning Education and Research (24) 2, 907-913.

Zürn, M. 2009. „Governance in einer sich wandelnden Welt - eine Zwischenbilanz“, in Perspektiven der Governance-Forschung, hg. v. E. Grande und S. May. Baden-Baden: Nomos, 61-76. 
Open Access Dieser Beitrag wird unter der Creative Commons Namensnennung 4.0 International Lizenz (http://creativecommons.org/licenses/by/4.0/deed.de) veröffentlicht, welche die Nutzung, Vervielfältigung, Bearbeitung, Verbreitung und Wiedergabe in jeglichem Medium und Format erlaubt, sofern Sie den/die ursprünglichen Autor(en) und die Quelle ordnungsgemäß nennen, einen Link zur Creative Commons Lizenz beifügen und angeben, ob Änderungen vorgenommen wurden.

Die in diesem Beitrag enthaltenen Bilder und sonstiges Drittmaterial unterliegen ebenfalls der genannten Creative Commons Lizenz, sofern sich aus der Abbildungslegende nichts anderes ergibt. Sofern das betreffende Material nicht unter der genannten Creative Commons Lizenz steht und die betreffende Handlung nicht nach gesetzlichen Vorschriften erlaubt ist, ist für die oben aufgeführten Weiterverwendungen des Materials die Einwilligung des jeweiligen Rechteinhabers einzuholen.

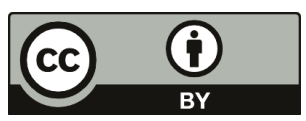

